\section{Epidemiologia da leishmaniose tegumentar na Comunidade São João, Manaus, Amazonas, Brasil}

\author{
Epidemiology of tegumentary leishmaniasis \\ in São João, Manaus, Amazonas, Brazil
}

Jorge Augusto de Oliveira Guerra 1,2 Jéfferson Augusto Santana Ribeiro 2 Leíla Inês de Aguiar Raposo da Camara Coelho 1,2 Maria das Graças Vale Barbosa 1,3,4 Marcilene Gomes Paes 1

\footnotetext{
1 Gerência de Leishmanioses, Fundação de Medicina Tropical do Amazonas, Manaus, Brasil.

2 Faculdade de Ciências da Saúde, Universidade Federal do Amazonas, Manaus, Brasil.

3 Centro Universitário Nilton Lins, Manaus, Brasil.

4 Programa de Pos-graduação em Medicina Tropical Universidade do Estado do Amazonas, Manaus, Brasil.

Correspondência Jorge A. O. Guerra Gerência de Leishmanioses, Fundação de Medicina Tropical do Amazonas. Av. Pedro Teixeira 25, Manaus, $A M$ 69040-000, Brasil. jorge@fmt.am.gov.br
}

\section{Abstract}

In Manaus, Amazonas State, Brazil, the degree of individual exposure to leishmaniasis is related to disorganized land occupation. In order to evaluate predisposing factors for an outbreak, confirm the parasitological diagnosis, treat patients, and assess etiological agents, reservoirs, and vectors, a 12-month study was conducted in Manaus in a community located along the BR-174 federal highway. Some 451 individuals were studied, among whom 17 cases of American tegumentary leishmaniasis (ATL) were diagnosed (six women and 11 men). Age varied from one to 64 years. Eleven patients had from one to three lesions. As for reservoirs, three opossums were captured. No hemoflagellates were found in the blood tests. Lutzomyia umbratilis was the predominant vector species captured. Many ATL patients were engaged in activities that exposed them to Leishmania vectors. Some patients may have been infected in the household and peridomiciliary environments. The epidemiological profile of ATL in this community is similar that of other foci in the region. This case series characterizes ATL as an endemic local public health problem.

Cutaneous Leishmaniasis; Leishmania; Disease Outbreaks

\section{Introdução}

A leishmaniose é um problema de saúde pública mundial e considerada pela Organização Mundial da Saúde 1 como uma das cinco doenças infecto-parasitárias endêmicas de maior relevância. As leishmanioses estão amplamente distribuídas nas Américas, estendendo-se desde o sul dos Estados Unidos ate o norte da Argentina.

No Brasil, apresenta-se em franco crescimento, tanto em magnitude como em expansão geográfica, observando-se a coexistência de um duplo perfil epidemiológico, expresso pela manutenção de casos oriundos dos focos antigos ou de áreas próximas a eles e pelo aparecimento de surtos epidêmicos associados a fatores como o acelerado processo de expansão das fronteiras agrícolas, a implantação de áreas de garimpos, a construção de estradas, e processo de invasão na periferia das cidades, entre vários outros. No período de 1985 a 1999, foram registrados no país 388.155 casos autóctones de leishmaniose tegumentar americana. $\mathrm{O}$ coeficiente de detecção de leishmaniose tegumentar americana no Brasil aumentou de 10,45/ 100 mil habitantes para 18,63/100 mil habitantes, com a melhoria do fluxo de informação 2 .

De acordo com dados do Departamento de Informática do SUS (DATASUS) ${ }^{3}$, são registrados no período de 1999 a 2003, cerca de 33.872 casos de leishmaniose tegumentar americana 
por ano no Brasil. A doença é registrada em todos os estados, observando-se sua expansão em amplitude e número de casos com 169.361 casos. A Região Sul apresenta o menor número, com 3.952 e a Região Norte o maior, com 61.339 no período descrito, e taxas de incidência por 100 mil habitantes entre 114,8 e 163,5 , para a Região Norte. No Amazonas, foram registrados 12.005 casos para o mesmo período, com taxas médias de incidência de 86,77 , sendo que no ano de 2003 esta taxa foi de 121,03 com 3.174 casos, $60,18 \%$ no Município de Manaus.

Na Amazônia, a leishmaniose tegumentar americana está agrupada sob três diferentes formas clínicas: leishmaniose cutânea, leishmaniose cutânea difusa e leishmaniose mucosa. No Estado do Amazonas a leishmaniose cutânea é a de maior predomínio, principalmente na região de Manaus 4,5.

No Amazonas, a leishmaniose tegumentar americana é primariamente uma zoonose 5, com ciclo de transmissão ocorrendo entre os flebotomíneos e os animais silvestres. Na maioria das vezes, o homem se infecta ao alterar o ambiente interpondo-se ao ciclo silvestre ao penetrar nesse ecossistema 6 .

Dentro do município, e principalmente na periferia da cidade de Manaus, o grau de exposição dos indivíduos acometidos está relacionado diretamente a processos de ocupação desordenada ou às chamadas invasões. Dessa forma, geralmente a leishmaniose tegumentar americana ocorre em lugares de assentamentos populacionais recentes e relacionada a desmatamentos 7,8 em populações próximas a áreas de floresta primária, onde a Leishmania (Viannia) guyanensis é enzoótica, 4 e em decorrência da construção de habitações desordenadas e sem qualquer planejamento, os indivíduos ficam expostos aos vetores.

É provável que a transmissão também ocorra no intra e no peridomicílio na região de Manaus, primeiro pela proximidade com as áreas de floresta 9 , pois grande parte das casas em assentamentos populacionais recentes é construída a menos de $100 \mathrm{~m}$ da orla da floresta e os indivíduos são alcançados pelo raio de ação dos vetores, que chegam às casas também atraídos pela luz 10; outro motivo é que sua fonte de repasto sangüíneo, que era feita nos animais silvestres, fica mais escassa pela presença humana, entretanto essa transmissão parece ser transitória, pois uma vez completada a urbanização a transmissão cessa. Paes et al. 9, em estudo em bairro de implantação antiga na cidade de Manaus, observaram um novo surto de leishmaniose tegumentar americana no qual 9,8\% dos casos estudados eram crianças menores de cinco anos, sugerindo transmissão no intra e peridomicílio. Andrade 10, em estudo realizado na Cidade de Deus, bairro da periferia de Manaus, localizado imediatamente à Reserva Florestal Adolpho Ducke, verificou a ocorrência de vários casos de leishmaniose tegumentar americana na rua que tem limite com a reserva.

Dentre os possíveis reservatórios silvestres da L. (V.) guyanesis, à preguiça (Choloepus didactylus) tem sido atribuído o papel principal na Amazônia brasileira 6 e em alguns locais da Guiana Francesa 11 nas áreas de floresta primária. Também implicado como importante reservatório silvestre está o Tamandua tetradactyla (tamanduá), e secundariamente temos Proechimys sp. (rato silvestre) e o Didelphis marsupialis (gambá) 5,6,12. Os parasitas podem ser encontrados na pele e nas vísceras desses animais 13,14, embora seu encontro não seja fácil.

O D. marsupialis adquire especial importância em áreas de floresta alteradas pelo homem 6,13,14 com uma taxa de infecção na região de Manaus de mais de $20 \% 12$. Este animal se alimenta em refugos domésticos nos limites da floresta próximos às casas, 5 ocorrendo constante circulação entre o meio florestal, onde se infecta, e o ambiente humano, onde serve de fonte de infecção para os flebótomos ali presentes 10 , estabelecendo possivelmente, dessa forma, um elo entre o ciclo silvestre e o peridomicílio.

A transmissão da leishmaniose tegumentar americana na região de Manaus é atribuída às Lutzomyia umbratilis e Lutzomyia anduzei, considerados, respectivamente, vetores principal e secundário da $L$. (V.) guyanensis, o parasito prevalente na etiologia da leishmaniose tegumentar americana/leishmaniose cutânea na região 15. No estudo de Naiff et al. 4, das espécies de Leishmania identificadas de 65 indivíduos portadores de leishmaniose tegumentar americana da região amazônica brasileira, a $L$. (V.) guyanensis foi encontrada em $83 \%$ dos casos, dos quais, $91 \%$ apresentavam lesão cutânea.

A outras espécies também é atribuída a etiologia de casos de leishmaniose cutânea na Amazônia, tais como L. (L.) amazonensis, L. (V.) naiffi, L. (V.) lainsoni, L. (V.) shawi, L. (V.) braziliensis e $L$. (V.) lindenbergi 16 .

Maior número de casos é registrado no período das chuvas (outubro a maio) 17,18 quando há aumento da densidade de vetores.

Durante o atendimento ambulatorial realizado na Fundação de Medicina Tropical do Amazonas (FMTAM), observou-se que alguns casos de leishmaniose tegumentar americana procediam do Km 4 da BR-174, localidade denominada São João. Essa comunidade possui cerca 
de 450 famílias. Como é uma área ainda em formação, não possui estruturas elementares de saúde: é um bairro sem saneamento básico, rede de esgotos e encanamento de água, e há asfalto apenas na rua principal.

A malária era o mais grave problema de saúde local, mas ocorria em áreas focais. A leishmaniose tegumentar americana parecia ser um problema de saúde importante na população naquela ocasião, e, portanto, merecedora de atenção e orientação visando a seu controle.

Este estudo teve como objetivos avaliar fatores predisponentes à gênese do surto de leishmaniose tegumentar americana; analisar a doença humana em seus aspectos sociais e econômicos e sua implicação na gênese do surto; confirmar o diagnóstico parasitológico e tratar os doentes; capturar e identificar prováveis reservatórios silvestres da leishmaniose tegumentar americana na área; e orientar e aplicar medidas preventivas.

\section{Metodologia}

O estudo foi desenvolvido no período de agosto 2001 a julho de 2002. A área de estudo é localizada em zona rural do Município de Manaus, Km 4 da BR-174, no sentido Manaus-Boa Vista. O acesso é asfaltado e pela rodovia de mesmo nome, e o assentamento está organizado.

A maioria das habitações é de madeira com cobertura de zinco ou amianto. Grande parte da população utiliza água de poços ou de fontes naturais tipo cacimbas. As condições de assistência à educação e saúde ainda são precárias. Existe uma escola e um Posto de Saúde funcionando onde um médico atende a cada 15 dias.

Foi feita entrada quinzenal na área, por médico, técnicos da Gerência de Leishmanioses da FMTAM (GLEISH/FMTAM) e os participantes do projeto para controle dos pacientes em tratamento e esclarecimento diagnóstico (por biópsia) de casos suspeitos não confirmados pela escarificação. Casos que necessitaram de esclarecimento diagnóstico, ou acompanhamento diferenciado, foram encaminhados à FMTAM para atendimento ambulatorial.

Nos pacientes com lesões suspeitas foi feita coleta da linfa-dérmica das bordas das lesões pela técnica da escarificação, para confirmação diagnóstica e tratamento. Também foram feitas biópsias das lesões de pacientes para semeadura em cultura (NNN ou agar sangue e Schnneider's) e inoculação em "hamster" para, e no sucesso do isolamento, identificar as cepas de Leishmania prevalentes na área.
Os casos positivos na lâmina foram acompanhados por médico da GLEISH/FMTAM para tratamento específico, considerando as condições do doente (outras doenças, idade, peso, gravidez etc.). Os esquemas de tratamento foram prescritos conforme orientação do Ministério da Saúde do Brasil. As pacientes grávidas com leishmaniose tegumentar americana só fariam os chamados tratamentos específicos após o parto, porém foram acompanhadas durante a gravidez por médicos da GLEISH/FMTAM.

$\mathrm{O}$ resgate dos doentes para controle clínico foi feito por agentes de saúde locais, em colaboração com a Secretaria Municipal de Saúde de Manaus, inclusive com a utilização das instalações do posto médico para atendimento de pacientes.

As estratégias em relação aos animais silvestres foram realizadas com a colaboração da comunidade. Fez-se na área captura de animais silvestres suspeitos de serem reservatórios, com exposição de armadilhas nas periferias das casas e nas matas adjacentes a estas, nos limites de no máximo 300m dos domicílios humanos. O período de exposição de armadilhas foi de sete dias, durante três meses consecutivos no ultimo trimestre de trabalho.

Foi sacrificado, para exame de vísceras, um exemplar de cada grupo encontrado, sendo destes pesquisada a presença de formas amastigotas de Leishmania em baço, fígado, linfonodos glândulas anais e pele, sendo feito exame histopatológico corado pela hematoxilina eosina, de todo este material, além de imprint para exame direto corado pelo Giemsa ou panótico; os demais, após examinados, foram marcados e soltos. Os animais foram avaliados para alterações de pelagem ou lesões suspeitas, coletas de amostras de sangue para avaliar hemoflagelados em culturas que, se positivas, seriam também inoculadas em "hamsters" na tentativa de isolamento e caracterização das cepas utilizando-se a técnica de anticorpos monoclonais.

Todos os animais possuíam uma ficha individual para registro de seus caracteres, e foram mantidos em cativeiro de acordo com as normas de manuseio de animais silvestres pelo menor tempo possível (apenas o necessário para coleta dos exames) na GLEISH.

A permissão do Instituto Brasileiro do Meio Ambiente e dos Recursos Naturais Renováveis (IBAMA) para manuseio dos animais silvestres, licença número 40/2002, foi concedida a partir de 10 de abril de 2002.

Em relação às medidas preventivas, foram realizadas orientações a todos que eram atendidos no posto sobre a forma de adquirir leis- 
hmaniose tegumentar e sobre a importância de quando necessário desmatar, não habitar de imediato e evitar penetrar na mata nos horários de maior atividade dos vetores.

O estudo foi desenvolvido de acordo com os requisitos do Conselho Nacional de Saúde, Resolução 196/96, sendo aprovado pelo comitê de ética em pesquisas envolvendo seres humanos da FMTAM.

Em projeto paralelo, desenvolvido pela Subgerência de Entomologia da FMTAM, foram realizadas coletas de insetos nas áreas de mata e peridomicílio para avaliar a densidade e diversidade de espécies de flebotomíneos na área.

Os flebotomíneos foram coletados durante 12 meses consecutivos, cinco dias a cada mês, em áreas de mata e no domicílio (extra, peri e intradomicílio) da comunidade, por meio de coletas manuais utilizando-se rede entomológica "puçá", capturadores manuais, armadilhas de luz CDC (estrato de $1 \mathrm{~m}$ ) e armadilhas Disney.

As coletas manuais no intradomicílio e peridomicílio foram realizadas em dois dias, durante a semana de estudo, no horário de 18:00 às 21:00, em residências localizadas até $100 \mathrm{~m}$ da borda da floresta que rodeia a comunidade. As coletas na base de árvores foram realizadas do segundo ao quarto dia durante 3 horas diárias (8:00 às 11:00), em diversos pontos dentro da mata delimitada para execução do estudo.

As coletas com as armadilhas CDC na mata eram iniciadas às 17:00 do primeiro dia (tro- cando-se as bolsas às 08:00 do dia seguinte) e encerradas às 08:00 do quinto dia. No peridomicílio foram colocadas nas casas onde seriam realizadas as capturas noturnas.

As armadilhas Disney foram colocadas a partir do primeiro dia, trocando-se as bandejas untadas com óleo mineral diariamente às 08:00 e retiradas no quinto dia.

Após as coletas o material foi levado para o Laboratório de Entomologia da FMTAM, onde foram realizadas triagens, dissecações, montagem de lâminas e identificação das espécies, utilizando-se chaves dicotômicas.

\section{Resultados}

Durante o período de estudo atendeu-se 451 pacientes no ambulatório da Comunidade São João, o coeficiente de prevalência encontrado para essa comunidade foi de 3,06 para $10 \mathrm{mil}$ habitantes. Foram diagnosticados 17 casos de leishmaniose tegumentar americana, cuja distribuição mensal se encontra na Figura 1.

Em apenas um caso a Leishmania foi isolada e aguarda-se a identificação da cepa. Seis pacientes eram do sexo feminino e 11 do masculino. O paciente mais novo tinha um ano de idade e o mais velho, 64 anos. Seis casos tinham entre 20 e 29 anos (Tabela 1).

Os estudantes constituíram o grupo predominante em relação à ocupação com 4 casos, seguidos pelos agricultores com 3 casos (Tabela 2).

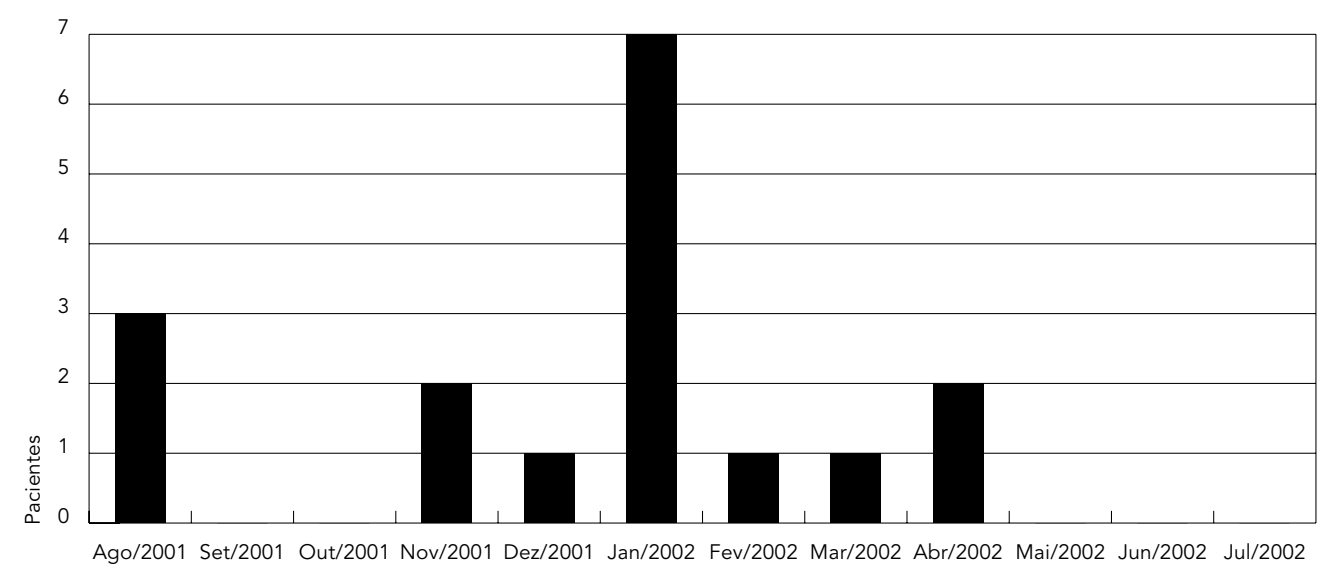


Onze pacientes tinham entre uma e três lesões, sendo as úlceras as mais freqüentes.

As leishmanides e infecção secundária foram observadas em sete doentes e a linfangite em quatro.

O diagnóstico dos casos de leishmaniose tegumentar americana foi feito com base em dados clínicos e confirmado pelo exame direto em 16 pacientes. Em um paciente o diagnóstico foi apenas clínico. O aspirado das lesões foi feito em quatro pacientes, pois nesta época o serviço de leishmaniose teve problemas de contaminação das culturas, conseguindo-se o isolamento da cepa em somente um deles, assim como desenvolvimento de lesões em "hamster", aguardando-se a identificação da cepa (Figura 2).

Os antimoniais foram utilizados para tratamento em 13 doentes; a pentamidina em dois; a azitromicina em um; uma paciente grávida teve involução espontânea das lesões. Cinco doentes tiveram recidiva das lesões e foram tratados novamente (Tabela 2).

Em relação aos animais silvestres, devido à demora da liberação da licença pelo IBAMA, houve tempo para se realizarem apenas dois períodos de exposição das armadilhas que foram colocadas na mata em locais diferentes em cada mês. Foram capturados três gambás $(D$. marsupialis). Estes animais foram examinados em busca de lesões sugestivas de leishmaniose tegumentar americana, que não foram encontradas, e tiveram sangue coletado e semeado em cultura. Um animal foi sacrificado, realizado exame histopatológico de suas vísceras e feita inoculação em "hamster". Os outros dois animais foram devolvidos a seu habitat natural. Também foi feita exposição de armadilhas no mês de janeiro de 2002, não havendo captura de animais. Os resultados das culturas dos três animais foram negativos, não se observou desenvolvimento de lesões nos "hamsters" e a histopatologia não mostrou sinais de infecção por Leishmania.

Foram coletados e identificados 4.104 espécimes de Psychodidae: Phlebotominae distribuídos em 49 espécies, dentre as quais destacam-se Lu. umbratilis 1.325 espécimes, representando $32,3 \%$ do material desse grupo, tendo sido coletada durante o ano inteiro, seguida de Lu. anduzei 820, Lu. (Trichophoromyia) eurypyga, 492, Lu. olmeca nociva 207, Lu. (Trichopygomyia) trichopyga 191, Lu. flaviscutellata 185 e Lu. (Psychodopygus) davisi, 154 (Tabela 3). Desse material chama-se a atenção para o registro de 24 espécimes de $L u$. umbratilis, um de $L u$. anduzei e um de Lu. olmeca nociva, coletados no peridomicílio.

\section{Discussão}

Com o final do ano e início do período das chuvas, observou-se aumento do número de casos de leishmaniose tegumentar americana, perfil característico àquele observado em diversos estudos $10,17,18$, condizente com os resultados en-

Tabela 1

Leishmaniose tegumentar americana na Comunidade São João, BR-174, Km 4, Manaus, Amazonas, Brasil. Distribuição de casos segundo a idade e o sexo.

\begin{tabular}{lccc}
\hline Idade (anos) & \multicolumn{2}{c}{ Número de pacientes } & Total \\
& Masculino & Feminino & \\
\hline$<10$ & 0 & 1 & 1 \\
$10-19$ & 1 & 3 & 4 \\
$20-29$ & 6 & 0 & 6 \\
$30-39$ & 4 & 1 & 5 \\
$>40$ & 0 & 1 & 1 \\
\hline
\end{tabular}

Leishmaniose tegumentar americana na Comunidade São João, BR-174, Km 4, Manaus, Amazonas, Brasil. Resposta ao tratamento administrado.

\begin{tabular}{|c|c|c|c|c|c|c|}
\hline \multirow{2}{*}{$\begin{array}{l}\text { Número de } \\
\text { pacientes }\end{array}$} & \multicolumn{3}{|c|}{ 1으 tratamento } & \multicolumn{2}{|c|}{ 2o tratamento } & \multirow[t]{2}{*}{ Total } \\
\hline & Medicação utilizada & Cura & Recidiva & Medicação utilizada & Cura & \\
\hline \multirow[t]{2}{*}{13} & Antimoniais & 9 & 4 & Antimoniais & 3 & 13 \\
\hline & & & & Anfotericina B & 1 & \\
\hline 2 & Pentamidina & 2 & 0 & & & 2 \\
\hline 1 & Azitromicina & 0 & 1 & Antimoniais & 1 & 1 \\
\hline 1 & Não tratado & 1 & 0 & & & 1 \\
\hline 17 & & 12 & 5 & & 5 & 17 \\
\hline
\end{tabular}


Figura 2

Leishmaniose tegumentar americana na Comunidade São João, BR-174, Km 4, Manaus, Amazonas, Brasil. Exames realizados nos doentes.

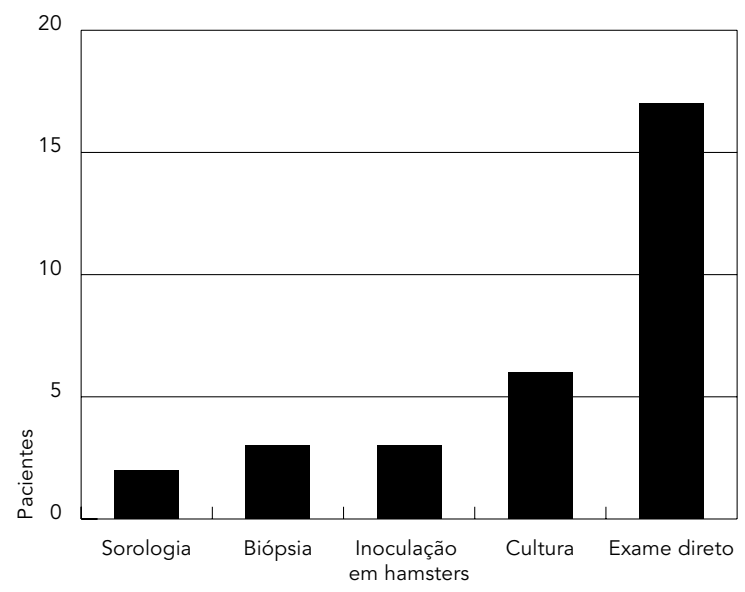

contrados neste trabalho. Boecken 19 verificou que o número de casos de leishmaniose tegumentar americana tem correspondência direta com o índice pluviométrico na região de Manaus.

Provavelmente esse fenômeno deva-se ao aumento da densidade dos vetores de leishmaniose tegumentar americana na base das árvores, na floresta, segundo verificado por Pajot et al. 11, na Guiana Francesa, onde a maioria dos casos ocorria no início do período chuvoso (dezembro e janeiro), quando as fêmeas infectadas de Lu. umbratilis eram excepcionalmente abundantes na base das árvores na floresta, fato mais uma vez comprovado pela captura de maior número de flebotomíneos nesse período, destacando-se a predominância da Lu. umbratilis.

A variação observada em relação à distribuição de casos entre as diversas faixas etárias apontam para a possibilidade de transmissão domiciliar, peridomiciliar e ocupacional. Grupos de menor idade, principalmente abaixo de seis anos, e idosos costumam ficar dentro ou ao redor das casas, indivíduos em fase produtiva, que se estima entre 20 e 50 anos, estão sob maior risco para aquisição da leishmaniose tegumentar americana devido às suas atividades laborativas na floresta, geralmente agricultura ou extrativismo. Boecken 19, em estudo realizado sobre o perfil epidemiológico da leishmaniose tegumentar americana em Manaus, veri- ficou que a maioria dos casos de leishmaniose tegumentar americana ocorreu em pacientes jovens do sexo masculino, com predomínio entre 20 e 29 anos.

Atualmente, tem-se observado uma mudança no padrão epidemiológico de transmissão das leishmanioses em diversos países da América do Sul, com uma importante domiciliação de vetores em países como a Venezuela, Peru, Bolívia e Brasil 20. Por este motivo o pensamento de que a leishmaniose tegumentar americana na América do Sul desapareceria como uma simples conseqüência da desflorestação tem sido revisto; estes exemplos constituem uma convincente evidência de que o aumento da domiciliação da transmissão de leishmaniose cutânea, já bem caracterizada em determinadas áreas no Brasil 21, está se expandindo na América Latina 20,22.

Uma parte significativa dos pacientes de leishmaniose tegumentar americana deste estudo tem atividades que os expõem aos vetores da Leishmania, como agricultura e trabalho em granjas. A maioria era do sexo masculino e tinha entre 20 e 29 anos. Houve um grupo de pacientes que possivelmente foi infectado no intra/peridomicílio, como uma criança de 12 meses 23 e uma senhora de 64 anos, e ainda uma casuística importante de donas-de-casa e estudantes. A aquisição da doença, nestes casos, pode ter ocorrido devido a flebótomos infectados, que foram atraídos em direção às casas por vários fatores, dentre eles a presença de animais domésticos, do homem, ou a presença de luz nas casas 16 , sugerindo a transmissão no peri/intradomicílio 9. Na Comunidade São João, as habitações são construídas próximas à orla da floresta e a atividade humana se desenvolve nesta ou em suas adjacências. Não há na literatura relatos de Lu. umbratilis, adaptado ao habitat peridoméstico 13; os indivíduos entram em contato com os vetores devido ao raio de alcance desses insetos a partir de seu habitat natural na floresta 10 . Em estudo epidemiológico realizado em Belo Horizonte, Minas Gerais, observou-se que $9 \%$ dos casos ocorrem na faixa etária entre zero e nove anos de idade 24 , porém na faixa de 5 a 15 anos a leishmaniose tegumentar americana também é freqüente, seja por penetração desses menores em áreas de mata em atividades de extrativismo vegetal, cultivo, desmatamento ou simplesmente por habitar próximo à floresta, entrando em contato com os vetores em seu ambiente natural, padrão comum no Município de Manaus 8,9,17.

Os habitantes da Comunidade São João costumam adentrar a mata para atividades extrativistas e mesmo buscar água em cacimbas ce- 
do pela manhã, período do dia em que parece haver maior chance de infecção por Leishmania, pois os flebotomíneos ainda são coletados em grande quantidade nas bases das arvores nas primeiras horas da manhã, conforme observado em estudos feitos na região 7,25. Assim, alguns dos pacientes estudados, que não possuíam atividade relacionada à mata, poderiam também ter se infectado ao adentrar a floresta para extrativismo, busca de água ou mesmo lazer.

Os reservatórios silvestres da leishmaniose tegumentar americana, reconhecidos na região de Manaus são: C. didactylus; T tetradactyla; Proechimys sp. e D. marsupialis, sendo este último um dos reservatórios mais importantes, pois tem o hábito de transitar no peridomicílio humano, estabelecendo um elo entre ciclo silvestre e peridomiciliar 6,10,12,13.

Guerra et al. 18, em trabalho com reservatórios em área periférica de Manaus, avaliaram 20 exemplares de $D$. marsupialis, dos quais se coletou material para cultura, obtendo isolamento de flagelados no sangue periférico, que, no entanto, não foram caracterizados como leishmânias. Na Comunidade São João é freqüente o relato de D. marsupialis na periferia das casas, sugerindo que estas, reconhecidamente reservatórios de leishmaniose, por seu caráter sinantrópico, estejam exercendo este papel em seu trânsito peridomicílio-mata, podendo ser encontrada alta taxa de infecção destes animais, explicável por uma diminuição no número dos reservatórios primários bem como aumento no número de gambás, atraídos pelo homem, nas áreas degradadas 26.

Em virtude do atraso na concessão da licença para manuseio de animais silvestres pelo IBAMA, retardou-se o início das atividades de captura. Dessa forma, poucos exemplares foram capturados, não permitindo adequada avaliação da taxa de infecção nos mesmos, bem como sua importância na cadeia de transmissão no local.

Com relação às características clínicas, na casuística de Romero et al. 27, pacientes com leishmaniose tegumentar americana por $L$. (V.) guyanensis apresentavam lesões mais numerosas e de menor diâmetro que os pacientes infectados por $L$. (V.) braziliensis. As lesões predominaram acima da cintura e o envolvimento linfático ocorreu em $61,8 \%$ dos pacientes. A ocorrência de indivíduos com numerosas lesões pode ser explicada pela ocorrência de picadas simultâneas por vários insetos infectados 4. Em nosso estudo, a maioria dos pacientes tinha entre uma e três lesões. A resposta ao tratamento é variável em diversas séries apresentadas, e os antimoniais foram eficientes em
Tabela 3

Leishmaniose tegumentar americana na Comunidade São João, BR-174, Km 4, Manaus, Amazonas, Brasil. Relação das espécies de flebotomíneos coletadas.

\begin{tabular}{|c|c|c|}
\hline Espécie & Total & $\%$ \\
\hline Brumptomyia pintoi (Costa Lima, 1932) & 4 & 0,10 \\
\hline Lutzomyia abonnenci (Floch \& Chassignet, 1947) & 3 & 0,07 \\
\hline Lu. amazonensis (Root, 1934) & 3 & 0,07 \\
\hline Lu. andersoni (Le Point \& Desjeux, 1988) & 2 & 0,05 \\
\hline Lu. anduzei (Rozeboom, 1942) & 820 & 20,00 \\
\hline Lu. antunesi (Coutinho, 1939) & 18 & 0,44 \\
\hline Lu. aragaoi (Costa Lima, 1932) & 27 & 0,66 \\
\hline Lu. ayrozai (Barreto \& Coutinho, 1940) & 1 & 0,02 \\
\hline Lu. b. barrettoi (Mangabeira, 1942) & 1 & 0,02 \\
\hline Lu. chagasi (Costa Lima, 1941) & 3 & 0,07 \\
\hline Lu. claustrei (Abonnenc, Léger \& Fauran, 1979) & 9 & 0,22 \\
\hline Lu. corossoniensis (Le Point \& Pajot, 1978) & 2 & 0,05 \\
\hline Lu. cuzquena (Martins, Llanos \& Silva, 1975) & 2 & 0,05 \\
\hline Lu. davisi (Root, 1934) & 154 & 3,75 \\
\hline Lu. dendrophyla (Mangabeira 1942) & 9 & 0,22 \\
\hline Lu. eurypyga (Martins, Falcão \& Silva, 1963) & 492 & 12,00 \\
\hline Lu. evangelistai (Martins \& Fraiha, 1971) & 1 & 0,02 \\
\hline Lu. flaviscutellata (Mangabeira, 1942) & 185 & 4,51 \\
\hline Lu. furcata (Mangabeira, 1941) & 26 & 0,63 \\
\hline Lu. geniculata (Mangabeira, 1941) & 12 & 0,29 \\
\hline Lu. Georgii (Freitas \& Barret, 2002) & 18 & 0,44 \\
\hline Lu. gomezi (Nitzulescu, 1931) & 55 & 1,34 \\
\hline Lu. h. hirsuta (Mangabeira, 1942) & 38 & 0,93 \\
\hline Lu. infraspinosa (Mangabeira 1941) & 23 & 0,56 \\
\hline Lu. inpai (Young \& Arias, 1977) & 7 & 0,17 \\
\hline Lu. longispina (Mangabeira, 1942) & 1 & 0,02 \\
\hline Lu. lutziana (Costa Lima, 1932) & 2 & 0,05 \\
\hline Lu. monstruosa (Floch \& Abonnenc, 1944) & 70 & 1,71 \\
\hline Lu. nematoducta (Young \& Arias, 1984) & 24 & 0,58 \\
\hline Lu. olmeca nociva (Young \& Arias, 1982) & 207 & 5,04 \\
\hline Lu. paraensis (Costa Lima, 1941) & 1 & 0,02 \\
\hline Lu. pilosa (Damasceno \& Causey, 1944) & 4 & 0,10 \\
\hline Lu. rorotaensis (Floch \& Abonnenc, 1944) & 79 & 1,92 \\
\hline Lu. ruii (Arias \& Young, 1982) & 8 & 0,19 \\
\hline Lu. scaffi (Damasceno \& Arouck, 1956) & 4 & 0,10 \\
\hline Lu. sericea (Floch \& Abonnenc, 1944) & 4 & 0,10 \\
\hline Lu. shannoni (Dyar, 1929) & 8 & 0,19 \\
\hline Lu. sordellii (Shannon \& Del Ponte, 1927) & 81 & 1,97 \\
\hline Lu. spathotrichia (Martins, Falcão \& Silva, 1963) & 12 & 0,29 \\
\hline Lu. squamiventris squamiventris (Lutz \& Neiva, 1912) & 5 & 0,12 \\
\hline Lu. triacantha (Mangabeira, 1942) & 72 & 1,75 \\
\hline Lu. trichopyga (Floch \& Abonnenc, 1945) & 191 & 4,65 \\
\hline Lu. trinidadensis (Newstead, 1922) & 1 & 0,02 \\
\hline Lu. trispinosa (Mangabeira, 1942) & 47 & 1,15 \\
\hline Lu. tuberculata (Mangabeira, 1941) & 7 & 0,17 \\
\hline Lu. ubiquitalis (Mangabeira, 1942) & 31 & 0,76 \\
\hline Lu. umbratilis (Ward \& Fraiha, 1977) & 1.325 & 32,30 \\
\hline Lu. walkeri (Newstead, 1914) & 1 & 0,02 \\
\hline Lu. williamsi (Damasceno, Causey \& Arouck, 1945) & 4 & 0,10 \\
\hline Total & 4.104 & 100,00 \\
\hline
\end{tabular}


$69,23 \%$ dos casos com apenas uma série de tratamento, diferente de Romero et al. 27, mas nossa pequena casuística não nos permite inferir maiores comentários a este respeito.

$\mathrm{O}$ registro das espécies $L u$. umbratilis e $\mathrm{Lu}$. anduzei, confirma o que já fora descrito quanto à sua predominância em regiões de florestas de terra firme na região da cidade de Manaus 14,15, 24 . A presença desses vetores no peri e intradomicílio e o fato de que as pessoas têm o acesso à mata, demonstra que os moradores da comunidade e os trabalhadores das propriedades particulares vizinhas estão expostos à transmissão da leishmaniose tegumentar americana, necessitando de um plano de vigilância e controle entomológico para combater as populações desses e de outros insetos vetores de doenças e, assim, contribuir para a melhoria da qualidade de vida desses moradores.

A leishmaniose é endêmica na área, e é um problema de saúde pública para a Comunidade São João.

A leishmaniose tegumentar americana na Comunidade São João apresenta um perfil clí-

\section{Resumo}

Em Manaus, Amazonas, Brasil, o grau de exposição dos indivíduos à leishmaniose está relacionado aos processos de ocupação desordenada. Para avaliar fatores predisponentes à gênese do surto, confirmar o diagnóstico parasitológico, tratar os doentes, avaliar agentes etiológicos, reservatórios e transmissores, realizou-se um estudo em Manaus, numa comunidade localizada no Km 4 da BR-174, durante 12 meses. Atendeu-se 451 indivíduos, dos quais foram diagnosticados 17 casos de leishmaniose tegumentar americana. Seis pacientes eram mulheres e 11 homens. A idade variou de um a 64 anos. Onze pacientes tinham entre uma e três lesões. Em relação aos reservatórios, três marsupiais foram capturados. Não foram encontrados hemoflagelados nos exames realizados. Dentre os insetos capturados, Lutzomyia umbratilis foi a espécie predominante. Grande parte dos pacientes de leishmaniose tegumentar americana tem atividades que os expõem aos vetores da Leishmania. Um grupo de pacientes foi infectado possivelmente no intra/peridomicílio. O perfil epidemiológico da leishmaniose tegumentar americana nessa comunidade é semelhante àquele observado em outros focos na região. Com essa casuística, a leishmaniose tegumentar americana é caracterizada como endêmica e como um problema de saúde pública local.

Leishmaniose Cutânea; Leishmania; Surtos de Doenças nico-epidemiológico semelhante a outros focos já descritos na região 9,10,17,18, observado em outras localidades em área rural do município e na periferia da cidade de Manaus, com moradias próximas das matas, pessoas adentrando e modificando essas matas e se expondo aos vetores.

D. marsupialis parece ser um animal importante na cadeia de transmissão da leishmaniose tegumentar americana no local, entretanto, são necessários mais estudos para estabelecer sua importância como reservatório da leishmaniose tegumentar americana nessa comunidade, como já demonstrado em outras localidades dentro do município.

A Lu. umbratilis foi a espécie prevalente na área, indicando, mais uma vez, seu papel como principal transmissor da leishmaniose tegumentar americana no município. No entanto, é importante a manutenção de medidas educativas dirigidas à comunidade, além de vigilância epidemiológica, entomológica e de reservatórios, visando ao controle dos casos.

\section{Colaboradores}

J. A. O. Guerra coordenou e atuou em todo o trabalho de pesquisa, elaborou, redigiu e fez as correções finais do artigo. J. A. S. Ribeiro participou de todo o trabalho de pesquisa, da elaboração e redação do artigo e da revisão bibliográfica. L. I. A. R. C. Coelho participou da parte de laboratório na pesquisa e da elaboração do texto. M. G. V. Barbosa participou na pesquisa, na redação geral do texto e discussão do trabalho na parte dos vetores e reservatórios. M. G. Paes participou de todo o trabalho de pesquisa e na elaboração da introdução e discussão do texto.

\section{Agradecimentos}

Os autores agradecem a Nelson Ferreira Fé pela identificação das espécies de flebotomíneos e auxílio nas coletas dos reservatórios. Gisele Santos, Flávio Fé e Elcimar Neves pelo auxílio nas coletas de flebotomíneos e Omarina Guerra pela revisão do texto. 


\section{Referências}

1. World Health Organization. Control of the leishmaniases. Geneva: World Health Organization; 1990. (Technical Report Series, 793).

2. Fundação Nacional de Saúde. Manual de controle da leishmaniose tegumentar americana. Brasília: Fundação Nacional de Saúde, Ministério da Saúde; 2000.

3. Ministério da Saúde. Rede internacional de informações para a saúde. Indicadores de morbidade e fatores de risco. http://tabnet.datasus.gov.br/ (acessado em 23/Out/2005).

4. Naiff MF, Cupolillo E, Naiff RD, Momen H, Barret TV, Grimaldi Jr. G. Leishmnaiose tegumentar americana na Amazônia: distribuição geográfica dos agentes etiológicos na região. Rev Soc Bras Med Trop 1999; 32 Suppl 1:243.

5. Barret TV, Senra MS. Leishmaniasis in Manaus, Brazil. Parasitol Today 1989; 5:255-7.

6. Lainson R. Our present knowledge of the ecology and control of leishmaniasis in the Amazon Region of Brazil. Rev Soc Bras Med Trop 1985; 18:47-56.

7. Barros MLB, Paes MG, Talhari S. Leishmaniose cutâneo-mucosa na Amazônia - estudo dos casos diagnosticados em Manaus no período de 1976 a 1980. An Bras Dermatol 1982; 57:153-4.

8. Talhari S, Arias JA, Cunha MGS, Naiff RD, Freitas RA, Barrett T. Leishmaniose no Estado do Amazonas - aspectos epidemiológicos clínicos e terapêuticos. An Bras Dermatol 1988; 63:433-8.

9. Paes MG, Fé NF, Coelho LIARC, Oliveira RS, Sousa JU, Teixeira MR, et al. Sobre a ocorrência de leishmaniose tegumentar em rua de bairro de implantação antiga na Cidade de Manaus (AM). Rev Soc Bras Med Trop 1998; 31:75.

10. Andrade SL. Leishmaniose tegumentar americana em área de ocupação recente na periferia da Cidade de Manaus, Estado do Amazonas, Brasil [Dissertação de Mestrado]. Rio de Janeiro: Departamento de Medicina Tropical, Instituto Oswaldo Cruz; 1997.

11. Pajot FX, Le Pont F, Gentile B, Besnard R. Epidemiology of leishmaniasis in French Guiana. Trans R Soc Trop Med Hyg 1982; 76:112-3.

12. Arias JR, Naiff RD, Miles MA, Souza AA. The opossum Didelphis marsupialis (Marsupialia: Didelphidae) as a reservoir host of Leishmania brasiliensis guyanensis in the Amazon Basin of Brazil. Trans R Soc Trop Med Hyg 1981; 75:537-41.

13. Lainson R, Shaw JJ. New World leishmaniasis the neotropical Leishmania species. In: Cox FEG, Krier JP, Wakelin D, editors. Topley \& Wilson's microbiology and microbial infections. v. 5. London: Arnold; 1998. p. 241-66.

14. Lainson R, Shaw JJ, Ready PD, Miles MA, Póvoa M. Leishmaniasis in Brazil: XVI isolation and identification of Leishmania species from sandflies wild mammals and man in north Pará State with particular reference to L. braziliensis guyanensis agent of "pian-bois". Trans R Soc Trop Med Hyg 1981; 75:530-6.

15. Arias JR, Freitas RA. On the vectors of cutaneous leishmaniasis in the Central Amazon of Brazil. Acta Amaz 1977; 7:507-27.
16. Silveira FT, Ishikawa EA, De Souza AAA, Lainson R. An outbreak of cutaneous leishmaniasis among soldiers in Belém, Pará State, Brazil caused by Leishmania (Viannia) lindenbergi n. sp. A new leishmanial parasite of man in the Amazon region. Parasite 2002; 9:43-50.

17. Guerra JAO, Barros MLB, Guerra MVF, Talhari S, Paes MG. Leishmaniose tegumentar no Município de Manaus - aspectos epidemiológicos. Rev Soc Bras Med Trop 1998; 31:172.

18. Guerra JAO, Souza AS, Lima AA, Dias CMF, Guerra MVF. Leishmaniose tegumentar americana (LTA) avaliação de dois anos de trabalhos com reservatórios em área periférica da Cidade de Manaus. Rev Soc Bras Med Trop 2001; 34:220.

19. Boecken GH. New World tegumental leishmaniasis in Brazil: with special reference to the situation of the disease in Manaus Central Amazon Basin [Dissertação de Mestrado]. London: University of London; 1996.

20. Campbell-Lendrum D, Dujardin JP, Martinez E, Feliciangeli D, Perez JE, Silans L, et al. Domestic and peridomestic transmission of American cutaneous leishmaniasis: changing epidemiological patterns present new control opportunities. Mem Inst Oswaldo Cruz 2001; 96:159-62.

21. Brandão Filho S, Campbell-Lendrum D, Brito MEF, Shaw JJ, Davies CR. Epidemiological surveys confirm an increasing burden of cutaneous leishmaniasis in north-east Brazil. Trans R Soc Trop Med Hyg 1999; 93:488-94.

22. Desjeux P. The increase in risk factors for leishmaniasis worldwide. Trans R Soc Trop Med Hyg 2001; 95:239-43.

23. Ampuero J, Urdaneta M, Macêdo VO. Factores de riesgo para la transmisión de leishmaniasis cutánea en niños de 0 a 5 años en un área endémica de Leishmania (Viannia) braziliensis. Cad Saúde Pública 2005; 21:161-70.

24. Passos VMA, Falcão AL, Marzochi MCA, Gontijo CMF, Dias ES, Barbosa-Santos EGO, et al. Epidemiological aspects of american cutaneous leishmaniasis in a periurban area of the metropolitan region of Belo Horizonte, Minas Gerais, Brazil. Mem Inst Oswaldo Cruz 1993; 88:103-10.

25. Paes MG. Estudo de quatro espécies de Lutzomyia França, 1924 (Diptera, Psychodidae) em área endêmica de leishmaniose tegumentar americana na periferia de Manaus [Dissertação de Mestrado]. Manaus: Fundação Universidade do Amazonas; 1991.

26. Lainson R. The american leishmaniasis: some observations on their ecology and epidemiology. Trans R Soc Trop Med Hyg 1983; 77:569-96.

27. Romero GAS, Guerra MVF, Paes MG, Macêdo VO. Comparison of Cutaneous Leishmaniasis due to Leishmania (Viannia) braziliensis and $L(V$.) guyanensis in Brazil: clinical findings and diagnostic approach. Clin Infect Dis 2001; 32:1304-12.

Recebido em 04/Jul/2005

Versão final reapresentada em 10/Jan/2006

Aprovado em 17/Jan/2006 